\title{
Wertorientierung und Medienpräferenz Jugendlicher
}

\author{
von Martin Hagenmaier
}

Während sich Medienpädagogen, Politiker und Eltern Sorgen darüber machen, wie Kinder und Jugendliche ein vermehrtes Programmangebot verkraften, sie mit Kabel und Video fertig werden, teilen Programmacher und Medienorganisatioren andere Sorgen, mit denen sie sich an die Medienforschung wenden. Die ARD/ZDF-Studie "Jugend und Medien", mit der Bertelsmann-Stiftung gemeinsam erarbeitet, spricht von der "Krise jugendlicher Medienkultur" angesichts der Tatsache, daß sich der bereits unterdurchschnittliche Medienkonsum von 1974 bis 1980 noch verringert habe.'

So fragen sich die Verantwortlichen von Presse, Rundfunk und Fernsehen, ob die Medienenthaltsamkeit eine jugendspezifische Erscheinung und allein durch attraktivere Konkurrenz im Freizeitbudget der Jugendlichen verursacht sei, oder ob Veränderungen im Medienkonsumverhalten Ausdruck dafür sind, daß die Zielgruppe "Jugendliche" vom Programmangebot verfehlt wurde, sind es doch in der Regel Erwachsene, die ein solches Angebot definieren.

So werden Veränderungen in der Jugendkultur für Medienschaffende interessant, Ansichten und Wertvorstellungen der Jugendlichen Gegenstand wissenschaftlicher Untersuchung im Hinblick auf die Frage, wie sich Einstellungen und Einstellungswandel, Werte und Wertewandel auf die Rezeption bestimmter Medien und ihrer Programme auswirkt. Zunächst soll eine Situationsbestimmung der Jugend versucht werden. Zwischen 'No-future'-Mentalität und Zuversicht werden eigene und gesellschaftliche Probleme erfahren und bewertet, werden Anliegen und Bedürfnisse sichtbar, Werthaltungen offenkundig. Es lassen sich Werttypen ermitteln und in ihrem Medienverhalten überprüfen.

\section{Die Situation}

Als "Tänzer" im Inneren des Eisbergs, als "zuckende Monade, eng gedrängt, jeder unendlich fern vom andern", so beschreibt Gerhard Staguhn seine Erfahrungen als "alter Esel" in einer Discothek und liefert damit zugleich eine Situationsbestimmung der Jugend von Heute. „Dort im heiligen Zentrum der Höhle tanzen sie also. Doch mehr als die Körper tanzt das Licht über ihnen. Dächte man es sich weg, so ergäbe das gleichmäßige Auf und Ab der Körper ein tristes, eisiges Bild von Beziehungslosigkeit nahe der Erstarrung. Das zuckende Scheinwerferlicht allein garantiert den Schein, daß hier so etwas wie Leben stattfinden könnte. Das Licht ist das Lebendigste hier" ${ }^{2}$ Der Wunsch nach Begegnung kommt gegen die "Diktatur des modischen 'Coolseins' nicht an ". Diese Jugend trägt am liebsten schwarz, als Ausdruck einer Stimmung, als Ausdruck eines Lebensgefühls. „No-future“ steht für Verweigerung, für Protest, weniger für Resignation.

Auf die Frage, ob sie die Zukunft der Gesellschaft eher zuversichtlich oder eher düster einschätzen, antworteten $198158 \%$ mit „eher düster", und $42 \%$ mit ,eher zuver-

Martin Hagenmaier ist Diplom-Theologe und lebt in Münster. 
sichtlich". Drei Jahre danach schätzten $46 \%$ die Zukunft der Gesellschaft eher düster, und $54 \%$ sie eher zuversichtlich ein. Die eigene Zukunft beurteilten 1984 nur $9 \%$ pessimistisch, dagegen $47 \%$ zuversichtlich.

Sind das die ersten Anzeichen für eine „Wende-Jugend“, für eine neue Generation von Jugendlichen, die optimistisch, oder vielleicht opportunistisch in die Zukunft blicken und die „no-future“-Mentalität hinter sich lassen? Arthur Fischer, der diese Zahlen im Rahmen der Shell-Studie ' $85^{3}$ vorstellte, warnt vor einer zu vereinfachten Sichtweise. Die differenziertere Fragestellung nach dem Eintritt bestimmter Ereignisse in der Zukunft offenbart nämlich sehr wohl eine Sensibilisierung für die Gefahren, mit denen die Gesellschaft in absehbarer Zukunft fertig werden muß. Die Aussage „Technik und Chemie werden die Umwelt zerstören" halten $74 \%$ der Jugendlichen für bestimmt oder wahrscheinlich in der Zukunft eintreffend, nur $2 \%$ weniger als 3 Jahre zuvor. Nur $33 \%$ sind der Meinung, daß es gelingen wird, die Umweltprobleme zu lösen. Die wirtschaftliche Entwicklung schätzen $65 \%$ skeptisch ein, und $74 \%$ erwarten eine noch höhere Arbeitslosigkeit. ${ }^{4}$ Dabei sind die eher pessimistisch in die Zukunft blikkenden Jugendlichen stärker an einer Veränderung der Situation interessiert. Sie neigen zu politisch alternativem Handeln, nehmen an Protestveranstaltungen teil und favorisieren „die Grünen ", während die eher optimistischen Jugendlichen die etablieten Parteien bevorzugen, tendentiell unpolitischer sind und in ihrer Freizeit lieber joggen oder tanzen gehen. Wer mit der gesellschaftlichen Situation zufrieden ist und für sich eine ungefährdete persönliche Zukunft erwartet, hält ein politisches Engagement für weniger notwendig

\section{Probleme und Bedürfnisse}

Welche Probleme stehen für die Jugendlichen im Vordergrund? Die Arbeitslosigkeit wird an erster Stelle und von $78 \%$ der Jugendlichen als sehr großes Problem benannt. Sie stimmen dabei in der Beurteilung mit den Erwachsenen überein, was darauf hindeutet, daß , weniger persönliche Problemdefinitionen, basierend auf eigener Lebenserfahrung", als vielmehr „Prozesse gesellschaftlicher Konsensbildung, maßgeblich

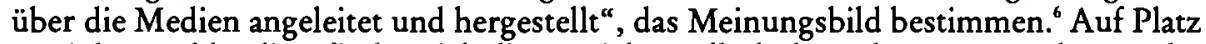
zwei der Problemliste finden sich die Bereiche „Alkohol“ und „Drogen“, die von der Hälfte der befragten Jugendlichen wie Erwachsenen als großes Problem eingestuft werden. Jeweils ein Drittel nennen Leistungsdruck oder Probleme in Schule und Ausbildung belastend. Von $15-20 \%$ als großes Problem empfunden werden Schwierigkeiten im individuellen und freizeitlichen Bereich: Reizüberflutung, Einsamkeit, fehlende Kontakte, Langeweile und die Unfähigkeit, die Freizeit sinnvoll zu gestalten. Keine Vorbilder mehr zu haben, geringen politischen Einfluß oder zu wenig Geld zu bekommen, macht den Jugendlichen noch am wenigsten Verdruß.' Doch müssen die genannten Problembereiche nicht unbedingt auf desolate Verhältnisse hinweisen. Während die ersten Ränge (Arbeitslosigkeit, Alkohol, Drogen) direkt gesellschaftliche Defizite ansprechen, die auch in der Offentlichkeit als solche diskutiert werden, so deuten die weiteren Problembereiche mehr auf die Werte hin, die für die Jugendlichen eine Rolle spielen. Auf die Frage, was sie zur Zeit am meisten beschäftige, geben $48 \%$ der Jugendlichen "Schule, Ausbildung und Beruf“ an, und $47 \%$ „Privatsphäre, Hobbies". "Andere Personen" (13\%), gesellschaftliche, politische, wirtschaftliche Probleme" $(8 \%)$ und "algemeine, globale Probleme" fanden nur am Rande das Interesse der Jugendlichen 8. Dabei gibt es im Altersablauf beträchtliche Schwankungen. Für die zwölf- bis dreizehnjährigen stehen Privatsphäre und Hobbies im Vordergrund. Ihre Bedeutung nimmt dann aber bis zum zwanzigsten Lebensjahr kontinuierlich ab, 
um danach wieder langsam anzusteigen. Umgekehrt drängt sich bis zum sechzehnten, siebzehnten Lebensjahr zunächst Schule, Ausbildung und Beruf ins Blickfeld, und zieht sich erst dann wieder zurück, wenn die meisten eine Ausbildung abgeschlossen haben und ins Berufsleben eingetreten sind. Die Bedeutung anderer Personen für das eigene Leben nimmt im Altersablauf langsam zu. Wenn nach den Bedürfnissen in der Freizeitgestaltung gefragt wird, steht „Interaktion “ an erster Stelle. Die zwölf- bis fünfzehnjährigen denken dabei in erster Linie an den Freundeskreis oder die Clique, während sich ab dem sechzehnten Lebensjahr dieser Kreis verkleinert, sich oft auf einen Partner beschränkt oder auf eine kleine Gruppe von „Menschen, die ich lieb habe $^{\text {". }}$. Bedürfnisse der Individuation nehmen den zweiten Rang ein. Das sind Möglichkeiten, -für sich selbst etwas zu tun, die Persönlichkeit weiterzuentwickeln, oder schöpferisch tätig zu sein. Erst dann folgen die klassischen Freizeitaktivitäten in Form von Bewegung (Sport, körperlicher Ausgleich), Eskapismus (Erholung, Ruhe, Ablenkung, Träume) und Lebensgenuß (Spaß, Abenteuer, Action, Ausflippen), wobei letzteres im Altersablauf noch an Bedeutung verliert. „Zusammengenommen ergibt sich das eher konventionelle Bild einer mehr individualistisch gesinnten heutigen Jugend, für die Sozialkontakte im engen Kreis, der private Freiraum, Selbstentfaltung und das Sich-Erholen-Können wichtige Freizeitanliegen sind, während offenbar eher extravertierte Aktivitäten und Genuß oder Erlebnisorientierung weniger bedeutsam zu sein scheinen ". ${ }^{10}$

\section{Werte und Wertewandel}

Nimmt sich der Wertewandel also nicht so eindeutig aus, oder ist die These, daß sich die Jugendlichen alternativ von der materialistisch geprägten Nachkriegsgeneration durch postmaterialistischen Idealismus, kommunikative Orientierung und gesellschaftliche Partizipation abheben wollen, schon durch eine neue Wende überholt? ${ }^{11}$ Wirtschaftskrise und Arbeitslosigkeit haben Ende der siebziger Jahre sicherlich das Interesse an einer materiell gesicherten Zukunft für die Jugendlichen in den Vordergrund gerückt, ohne daß sie sich aber darüber hinaus materielle Werte wie Luxus, Reichtum, Wohneigentum oder Auto zu eigen gemacht haben. Sie haben sich bei der Suche nach Arbeit und Beruf vielmehr von eigenem Interesse leiten lassen, weniger nach dem Verdienst, als nach der Möglichkeit gefragt, sich selbst in einem schöpferisch produktiven Tun zu verwirklichen.

Alte und neue Werte stehen oft nebeneinander, bilden eine Art widersprüchlicher Harmonie. ${ }^{12}$ Nur Minderheiten sind es, die die eine oder andere Lebensform sich radi$\mathrm{kal}$ zu eigen machen. So ist eine Mehrheit der Jugendlichen der Meinung, daß Jugendliche, aber auch Erwachsene sich „alte Werte “ wie gute Umgangsformen, Sparsamkeit, Ordnung, Pflichtgefühl, Fleiß und Zufriedenheit aneignen sollen. Umgekehrt

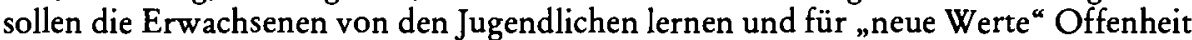
zeigen, neue Lebensformen finden, aus Sachzwängen ausbrechen, lockerer, spontaner leben, mehr auf andere Menschen eingehen und nicht nur an materielle Dinge denken. ${ }^{13} \mathrm{Zwar}$ lassen sich als soziale Trägergruppen für die neuen Werte die Jugendlichen und für die alten Werte die Erwachsenen ausmachen, die Verteilung und Gewichtung der Werte ist dennoch nicht gleich: Die Erwachsenen lehnen in stärkerem Maße die neuen Werte ab (zu $55 \%$ ) als die Jugendlichen dies mit den alten Werten tun (29\%). ${ }^{14}$

Eine differenziertere Erfassung der Wertprioritäten Jugendlicher hebt in den ersten zehn Rängen eindeutig Werte im Bereich der Arbeit und der sozialen Beziehungen hervor. Freunde haben, denen man vertrauen kann, lieben und geliebt werden und ein 
harmonisches Familienleben stehen ebenso hoch im Kurs wie der sichere Arbeitsplatz, eine gute Ausbildung, Erfüllung in der Arbeit und Erfolg im Beruf. Am Ende der Liste stehen materialistische, aber auch idealistische und post-materialistische Werte: Macht und Einfluß, Reichtum und Luxus, hohes Einkommen und Ansehen in der Gesellschaft werden ebenso gering geschätzt wie politische Überzeugung, Kunst und Kultur, Religion und Glaube. Im mittleren Bereich finden sich Freizeitwerte, Urlaub, Reisen, Unabhängigkeit und mit Auto, Motorrad, Mofa auch die dafür notwendige technische Mobilität. Im Altersablauf nehmen Werte der Sicherheitsorientierung und des privaten Glücks an Bedeutung zu, Werte der Berufszufriedenheit pendeln sich in einem mittleren Bereich ein, während die mehr hedonistischen Wertorientierungen eher zurückgehen. Materialistische, aber auch idealistische und postmaterialistische Werthaltungen bleiben auf einem unteren Niveau stabil. ${ }^{15}$

\section{Die Werttypen}

Personen mit überwiegend gemeinsamen Werthaltungen können zu Werttypen zusammengefaßt werden. In einer solchen Gruppe sind ein oder mehrere Wertfaktoren besonders ausgeprägt. Der Werttyp der „Yuppies“, der „Geld, Genuß, Beruf“ in den Mittelpunkt seines Lebens stellt, hat einen Anteil von $24 \% .{ }^{16}$ Für ihn sind Erfolge im Beruf ebenso wichtig wie materielle und hedonistische Werthaltungen. Einen Anteil von $22 \%$ hat der Typ „Familie, Sicherheit, Beruf“, für den das private Glück, aber auch materielle Sicherheit und Berufszufriedenheit zentrale Werte sind. Der Typ „Sicherheitsorientierung" wird von $14 \%$ der Jugendlichen verkörpert. Vor allem ältere männliche Jugendliche, Lehrlinge und Berufstätige legen Wert auf materielle und soziale Sicherheit. 12 \% der Jugendlichen und jungen Erwachsenen, davon viele mit höherem Bildungsniveau und Studenten, sind dem Typ „Idealismus, Postmaterialismus" zuzurechnen.' Zu dem Typ „privates Glück “ gehören vor allem Frauen und ältere, berufstätige Jugendliche. Sie machen einen Anteil von $9 \%$ aus. Familie und persönliche Geborgenheit stehen für sie im Vordergrund. Dagegen wird der Typ „Materialismus“ mit einem Anteil von $8 \%$ verstärkt von jüngeren und männlichen Jugendlichen vertreten. Eine ähnliche Zusammensetzung findet sich bei dem Typ „Hedonismus“. Mit $5 \%$ ist diese Gruppe Jugendlicher, die ein genußreiches Leben schätzen, am geringsten vertreten. ${ }^{18}$

Diese Werttypen lassen sich in ihrem Freizeit- und Medienverhalten beobachten. Sie geben Aufschluß darüber, wie eine Werthaltung einen Jugendlichen prägt und bestimmte Verhaltensweisen beeinflußt.

\section{Mediennutzung und Freizeitaktivitäten}

Jugendliche können werktags im Durchschnitt 5,5 Stunden für eigene Freizeittätigkeiten verwenden. Am Wochenende haben sie 7,5 bis 8,25 Stunden zur freien Verfügung. Während diese Zahlen in den letzten zehn Jahren relativ stabil blieben, so änderte sich doch der Zeitanteil, der außer Haus verbracht wurde, von $26 \%$ im Jahre 1974 auf nahezu $50 \%$ 1984. Die meisten nicht-medialen Freizeitaktivitäten werden in einem wöchentlichen Rhythmus wahrgenommen. Dabei steht das gesellige Beisammensein mit Freunden, Bekannten oder der Familie an erster Stelle, gefolgt von sportlichen Betätigungen und der Pflege eines Hobbies. Dagegen scheinen Veranstaltungen wie Kino-, Theater- oder Konzertbesuch aber auch Vereinsanlässe, Lesungen, Vorträge oder Weiterbildungskurse nur für eine Minderheit attraktiv zu sein. Von den Werttypen sind es vor allem die idealistisch und postmaterialistisch Orientierten, die 
überdurchschnittlich häufig Veranstaltungen besuchen, aber auch verstärkt einem Hobby nachgehen. Jugendliche, für die das "private Glück“ im Vordergrund steht, interessieren sich ebenfalls für Hobbies und wenden sich daneben der Geselligkeit im Kreis der Freunde oder Familie zu. Hedonistisch veranlagte Jugendliche finden an sportlich und spielerisch verbrachter Freizeit am meisten Gefallen, materialistisch gesinnte nehmen gern an Veranstaltungen teil, sind bei anderen aktiven Freizeitbeschäftigungen jedoch weniger vertreten.

Im medialen Freizeitbereich greifen die Idealisten und Postmaterialisten häufiger zu einem Buch oder zur Zeitung. Das Interesse am Buch teilen sie dabei mit Jugendlichen, die in ihrem Beruf aufgehen und in der Zufriedenheit mit ihrem Beruf ein zentrales Anliegen sehen, das Interesse an der Zeitung mit Jugendlichen, denen die Familie, Sicherheit und deshalb auch der Beruf am Herzen liegen. Der Fernsehkonsum ist dort am höchsten, wo privates Glück, Sicherheitsorientierung und Familie eine Rolle spielen. Wer dem Hedonismus zuneigt, findet sich sowohl unter den Fernsehzuschauern, als auch unter den Zeitschriftenlesern und räumt dazu dem Radio, Platten und Kassetten einen breiten Raum ein. Am wenigsten wird er Bücher und Zeitungen lesen $^{19}$

Insgesamt widmen sich Jugendliche 4 Stunden und 54 Minuten am Tag den verschiedenen Massenmedien. Die auditiven Medien nehmen mit $49 \%$ der Zeit einen breiten Raum ein. Dieser Medienkonsum hat aber eher sekundären Charakter, d. h. es werden daneben noch andere Tätigkeiten verrichtet. Das Radio kann sich dabei mit 40 \%gegenüber dem Gebrauch von Plattenspieler und Kassettenrecorder (zusammen 9\%) sehr gut behaupten. Mehr als ein Drittel der für Medien verwendeten Zeit gehört dem Fernsehen. Im Vergleich der letzten $10 \mathrm{Jahre}$ erhöhte sich die Zeit, in der Radio gehört wird, von 1974 bis 1980 und ging dann bis 1984 stark zurück. Mit dem Fernsehkonsum verhielt es sich umgekehrt: Bis 1980 verringerte sich das Fernsehinteresse, um danach wieder das alte Niveau zu erreichen. Die Sorgen und Ängste der Programmacher, das jugendliche Publikum nicht zu erreichen, sind also zumindest im Blick auf die allgemeinen Durchschnittswerte nicht begründet.

Im Altersablauf nimmt die Attraktivität des Radios mit dem Alter zu, die Einschaltminuten verdoppeln sich. Demgegenüber verringert sich die Zeit, in der Platten oder Kassetten gehört werden, um die Hälfte. Der Fernsehkonsum erreicht $z$ wischen dem zwanzigsten und dem vierundzwanzigsten Lebensjahr die niedrigsten Werte und unterliegt damit der Konkurrenz einer aktiven Freizeitgestaltung, die Veranstaltungsbesuche und geselliges Beisammensein bevorzugt. Das Interesse am Buch nimmt mit dem Alter ab, während die Zeitungslektüre an Bedeutung gewinnt.

Die durchschnittlichen Nutzungszeiten der einzelnen Medien sind zum Teil recht niedrig. Das liegt unter anderem daran, daß die Medien unterschiedliche Reichweiten haben. So liest nur jeder fünfte Jugendliche gerne Bücher. Wer sich aber dieser $\mathrm{Be}$ schäftigung verschreibt, verbringt damit 1 Stunde und 40 Minuten am Tag. Nur $5 \%$ der Befragten verfügen zu Hause über einen Videorecorder. Er läuft dann aber mehr als zwei Stunden täglich. Telespiele werden von denen, die über eine solche Möglichkeit verfügen, im Durchschnitt 1 Stunde und 20 Minuten gespielt. Wer gerne Radio hört, hat das Gerät gute zweieinhalb Stunden eingeschaltet, Platten- und Musikkassetten-Fans kommen auf zwei Stunden Einschaltzeit. Etwas weniger als zweieinhalb Stunden verbringen Fernsehzuschauer vor ihrem Gerät 20. Diese Nutzungszahlen, die sich nur an denjenigen Jugendlichen orientieren, die ein Medium tatsächlich ge- 
brauchen, kommen der Wirklichkeit etwas näher als die allgemeinen Durchschnittswerte. Vor allem lassen die Angaben im Bereich der Telespiele und des Videokonsums Schlüsse auf eine Zukunft zu, in der mehr Jugendliche über solche Geräte verfügen.

\section{Medienpräferenzen}

Welche Fernsehprogramme sind bei Jugendlichen beliebt, welche Musiksparten werden bevorzugt, welche Bücher werden gelesen?

Im literarischen Bereich steht die Unterhaltung nicht an erster Stelle. Berufliche Fachund Sachbücher, Lexika und Schulbücher bestimmen den täglichen Bedarf. Für die Schul- und Berufsausbildung ist das Buch immer noch das Medium Nummer eins. In der Belletristik belegen Humor und Satire, Krimi- und Spionage-Literatur die vorderen Ränge. Weibliche Leser bevorzugen Liebes-und Schicksalsromane, greifen gelegentlich zu Koch- und Hobbybüchern, und können sich auch an moderner Literatur und Biographien erfreuen, während männliche Jugendliche Science-Fiction und Abenteuer, Naturwissenschaft und Technik favorisieren. Eine höhere Schulbildung weitet das Interesse für klassische und moderne Litertaur, für Kunst und Kultur, sachliches und anspruchsvolles. ${ }^{21}$

Unter den Werttypen sind es die Idealisten und Postmaterialisten, die sich am ehesten für anspruchsvolle Literatur entscheiden, relativ häufig zu einem Sachbuch greifen und generell in allen Sparten zu Hause sind. Wer dem Hedonismus zuneigt, liest insgesamt recht wenig und bevorzugt Kinder- und Jugendbücher. Er ist der Trivialliteratur nicht abgeneigt, die auch sonst ihre Anhänger findet, so z. B. unter den Yuppies, die "Geld, Genuß, Beruf" erstreben, oder materialistisch eingestellten Jugendlichen. Wo Familie und Sicherheitsorientierung als zentrale Werte vorherrschen, sind besonders Ratgeberbücher gefragt. Berufszufriedenheit fördert die Nachfrage nach Fach- und Sachbüchern. ${ }^{22}$

Im musikalischen Bereich sind die Vorlieben recht eindeutig. Von über $60 \%$ der Jugendlichen wird internationale Pop- und Discomusik gerne oder sehr gerne gehört. Die breiteste Ablehnung erfährt Oper, Operette, Musical und Deutsche Volks- und Blasmusik. Darüber hinaus ist der Musikgeschmack sehr stark individualisiert und findet häufig ebensoviel Ablehnung wie Zustimmung. Soziodemographische Einflüsse lassen sich nicht leugnen. Frauen hören lieber leichte moderne Unterhaltungsmusik oder klassische Musik, Männer sind unter den Anhängern des Jazz, Heavy Metal und Hard Rock mehr vertreten. Im Altersablauf löst sich die starre Fixierung auf internationale Pop- und Rockmusik allmählich auf. Das Toleranzspektrum wird breiter. ${ }^{2}$

Von den Werthaltungen sind es hedonistische, an Geld, Genuß und Beruf orientierte Jugendliche, die moderner Popmusik den ersten Platz einräumen. Familie, Glück und Geborgenheit konvergieren mit einer Neigung zur leichten Unterhaltung oder zur klassischen Musik. Das stärkste Interesse für klassische Musik kommt jedoch von Jugendlichen mit idealistischer und postmaterialistischer Werthaltung, die auch unter den Jazzfreunden einen erhöhten Anteil haben.

Das Fernsehen erweist sich für die Jugendlichen als ein Medium der Unterhaltung, der aktuellen Information und im Besonderen auch der Musik. Spielfilme, Nachrichten und Musiksendungen belegen die ersten Ränge in der Beliebtheitsskala. Auch Serien und Krimis werden noch von einer Mehrheit der Jugendlichen regelmäßig oder häufig gesehen. Die weiteren Sparten finden ebensoviel Zustimmung wie Ablehnung. 
Schlußlicht bildet wie bei der Musik auch im Fernsehen die Oper, neben Musical, Operette und Volksmusik. Während die Nachrichten unter den regelmäßig gesehenen Sendungen den ersten Platz einnehmen, finden politische Magazine und Berichte kaum eine Resonanz. Dabei spielt das Alter eine wesentliche Rolle. Von den 12 bis 15jährigen Jugendlichen sehen rund ein Viertel regelmäßig oder häufig die Fernsehnachrichten. Im Alter zwischen sechzehn und neunzehn hat sich dieser Anteil verdoppelt und bei den 25 bis 29 jährigen verdreifacht. Insgesamt finden Informations- und Bildungsprogramme mit zunehmendem Alter mehr Beachtung. Ihr Interessentenkreis wird weiterhin von männlichen Jugendlichen mit höherem Bildungsniveau dominiert, idealistische und postmaterialistische Werthaltungen sind in der Überzahl. Dazu gehören ferner Leute, die überhaupt wenig fernsehen und viel lesen, und neben informativen Programmen auch für anspruchsvolle Unterhaltung zu haben sind.

Das Gegenstück dazu bilden leichte Unterhaltung, Alltags- und Familienserien. Sie werden von weiblichen Zuschauern, Jugendlichen mit Volksschulbildung und NichtBerufstätigen begünstigt, die in ihren Werthaltungen der Familie und dem privaten Glück den Vorzug geben. Harte Unterhaltung dagegen, Krimis, Western und Abenteuerfilme, sind eine männliche Domäne. Häufiges Fernsehen gehört ebenso zu diesem Zuschauertypus wie niederes Bildungsniveau und Wertorientierung in Richtung "Geld, Genuß, Beruf" und Materialismus.

Jugend- und Musiksendungen im Bereich der Rock- und Popmusik finden vor allem bei den Jüngeren mit hedonistischer Werthaltung Gefallen. Die speziell für Jugendliche produzierten Sendungen werden von einem Drittel aller Angesprochenen regelmäßig oder häufig rezipiert. Bei den unter 15jährigen liegt der Anteil doppelt so hoch, so daß diese Zielgruppe durchaus erreicht wird.

Werden die Jugendlichen gefragt, was zuviel oder zuwenig im Fernsehen angeboten werde, spiegeln sich in den Antworten die vorhandenen Nutzungsgewohnheiten. Vom Gängigen und Beliebten, Spiel- und Kinofilme, Jugend- und Musiksendungen, wird mehr gewünscht, Sendungen aus Politik und Wirtschaft werden dagegen abgelehnt. Je nach Alter und Bildung sind die Fernsehwünsche recht widersprüchlich: was dem Einen zuviel, ist dem Andern zuwenig. ${ }^{24}$

Fühlen sich Produzenten und Programmacher bei diesen Zahlen bestätigt, stimmen sie Medienpädagogen noch bedenklich. Da ist z. B. die Bildungsschere nicht zu übersehen. Der Gebildete liest Bücher und schaut wenig fern. Er liest keine Trivialliteratur und schaut sich im Fernsehen Sendungen aus dem Bereich der Bildung und Information oder Kultur an. Jugendliche mit niederem Bildungsniveau ziehen dagegen die Unterhaltungssparten des Fernsehens vor. Das Fernsehen scheint dennoch weniger dazu geneigt, Bildungslücken zu überbrücken, als vielmehr solche zu vertiefen. Liegt es am mangelnden jugendlichen Interesse für kulturelle, wirtschaftliche und gesellschaftliche Probleme, wenn solche Sendungen nicht angenommen werden, sind die Programmformen zur Vermittlung dieser Probleme ungeeignet, oder liegt es am Medium selbst, daß es seinem Image nach der Unterhaltung näher als der Bildung steht?

Werthaltungen spiegeln Lebensentwürfe wider. Die Einstellung zur Welt, zur Gesellschaft und zum Leben macht sich in der Medienrezeption bemerkbar. Die Nutzung eines Mediums hängt sicherlich von einem entsprechenden Angebot ab. Die Bedeutung aber, die dem einzelnen Menschen zukommt, der sich für das eine oder andere Programm oder eine alternative Freizeitgestaltung entscheiden muß, darf nicht übersehen werden. 


\section{Anmerkungen}

1 Jugend und Medien, Media Perspektiven 6, Frankfurt 1986, 9.

2 Gerhard Staguhn, Tänzerim Innern des Eisbergs, in: Frankfurter Rundschau, 02.08.86, ZB 3.

3 Arthur Fischer, Zukunft und Politik, in: Jugendliche und Erwachsene '85, Jugendwerk der Deutschen Shell, Opladen 1985, Bd. I, $106 \mathrm{ff}$.

4 ebd. $116 \mathrm{ff}$.

5 ebd. 121.

6 Jürgen Zinnecker, Beziehungen zwischen jüngerer und älterer Generation im Urteil von Jugendlichen und Erwachsenen, in: Jugendliche und Erwachsene '85, a.a.O., 95.

7 ebd., 96.

8 Media Perspektiven, 41.

9 ebd., 42.

10 ebd., 43.

11 ebd., 46; vgl. R. Inglehart, The silent resolution, Princeton 1977, vgl. Roman Bleistein, Zur Lebenssituation junger Menschen, in: Stimmen der Zeit 7, 86, 466.

12 ebd., außerdem: Die verunsicherte Generation: Jugend und Wertewandel, SINUS-Instituh, Opladen 1983, 16.

13 Shell, a.a.O., 77.

14 ebd., 79.

15 Media Perspektiven, a.a.O., 47.

16 ebd., 49.

17 ebd., 51.

18 ebd., 53, 56.

19 ebd., $89 \mathrm{ff}$.

20 ebd., $141 \mathrm{ff}$.

21 ebd., $105 \mathrm{ff}$.

22 ebd., 115.

23 ebd., 154-157.

24 ebd., 145-153.

\section{Summary}

There are signs making one fear that the adults of tomorrow cannot be reached (any more) with the communication forms used today. Recent structural data on the development of the youth and of young persons caused the author to relate the youth's awareness of problems and their needs, their standards of value and their moral concept to the youth's leisure-time activities and their use of media. In the form of a brief literary study follow interrelated topics that require more detailed and thorough examination.

\section{Résumé}

On peut observer les signes qui amènent à la crainte que les adultes de demain ne seront pas (plus) à atteindre avec les formes de communications actuelles et courantes. Les plus nouvelles structures de données au developpement de la jeunesse et des jeunes ont occasionnées l'auteur de mettre 
en relation le problème de prise de conscience, la tenue des besoins, leurs critères de mesure, leur orientation de valeurs et le comportement des jeunes pendant leur temps libre comme aussi l'utilisation de massmedia. On a mis comme suivant en évidence dans une forme résumée d'une étude litéraire un champs de thèmes qui sera encore examiné plus exactement et plus profundement.

\section{Resumen}

Hay indicios por los cuales es de temer que (ya) no se podrá alcanzar los adultos de manana con las formas de comunicación usadas hoy. Los recientes datos estructurales sobre el desarrollo de la juventud y de los jóvenes causaron el autor que relacionara la conciencia de problemas y las necesidades de los jóvenes, sus normas y sus valores con el juvenil aprovechamiento del tiempo libre y el uso de los medios. En forma de un breve estudio de la literatura presenta a continuación temas que requieren un estudio más detallado y profundo. 